\title{
Invasive Aspergillosis: Performance of New and Established Diagnostic Approaches with Same-Day Blood and Bronchoalveolar Lavage -- a Prospective Cohort Study
}

S. Heldt'1,2, J. Prattes ${ }^{2,3}$, S. Eigl ${ }^{1}$, B. Spiess ${ }^{4}$, H. Flick¹, J. Rabensteiner ${ }^{5}$, G. Johnson ${ }^{6}$, F. Prüller ${ }^{5}$, A. Wölfler ${ }^{3,7}$, T. Niedrist ${ }^{5}$, T. Boch ${ }^{4}$, P. Neumeister ${ }^{7}$, H. Strohmaier ${ }^{8}$, R. Krause ${ }^{2,3}$, D. Buchheidt ${ }^{4}$, and M. Hoenig|1,2,3,9

${ }^{1}$ Division of Pulmonology, Medical University of Graz, Austria; ${ }^{2}$ Section of Infectious Diseases and Tropical Medicine, Medical University of Graz, Austria; ${ }^{3} \mathrm{CBmed}$ - Center for Biomarker Research in Medicine, Graz, Austria; ${ }^{4}$ Department of Hematology and Oncology, Heidelberg University Mannheim University Hospital, Germany; ${ }^{5}$ Clinical Institute of Medical and Chemical Laboratory Diagnostics, Medical University of Graz, Austria;

${ }^{6}$ OLM Diagnostics, Newcastle-upon-Tyne, United Kingdom; ${ }^{7}$ Division of Hematology, Medical University of Graz, Austria; ${ }^{8}$ Center for Medical Research, Medical University of Graz, Austria; ${ }^{9}$ Division of Infectious Diseases, Department of Medicine, University of California-San Diego, USA;

Objectives: Aspergillus spp. and other molds have been shown to induce elevated levels of several cytokines. It remains unknown whether these cytokines hold value for clinical routine and enhance diagnostic performances of established and novel biomarkers/ molecular tests for IA and other IMI.

Aims: To determine the diagnostic potential of a number of cytokines, as well as established and emerging tests for IA and IMI in patients with underlying hematological malignancies in a setting that uses mold-active prophylaxis.

Methods: This cohort study included 106 prospectively enrolled (2014-2017) adult cases with underlying hematological malignancies and suspected pulmonary infection undergoing bronchoscopy. Serum samples were collected within 24 hours of BALF sampling. Both, serum and BALF samples were used to evaluate diagnostic performances of the Aspergillus-specific LFD, Aspergillus PCR, GM, BDG, and a bundle of cytokines for IA and IMI, classified according to the revised EORTC/MSG criteria.

Results: Among the 106 cases, 11 had probable IA, 32 possible IA (of which 7 had probable IMI), and 63 no evidence for IA. Combinations of serum IL-8 with either BALF LFD (sensitivity $100 \%$, specificity $94 \%$ ) or BALF PCR (sensitivity $91 \%$, specificity $97 \%$ ) were highly sensitive and specific for differentiating probable IA from no IA. Serum IL-8 showed also potential for differentiating patients with possible/probable IMI from those without IMI (AUC 0.641, $p<0.014$ ), while serum and BALF GM (AUC 0.607, p>0.05 and AUC $0.503, p>0.05$, respectively) and serum BDG (AUC 0.514, $p>0.05$ ) did not.

Conclusion: Our study indicates that serum IL-8 testing may be a valuable addition to clinical routine for diagnosing IA in high risk patients who receive mold-active antifungals.

Figure 1: ROC curve analysis for IL-6 \& IL-8 in serum \& BALF samples for cases with probable IA vs. no evidence for IA.

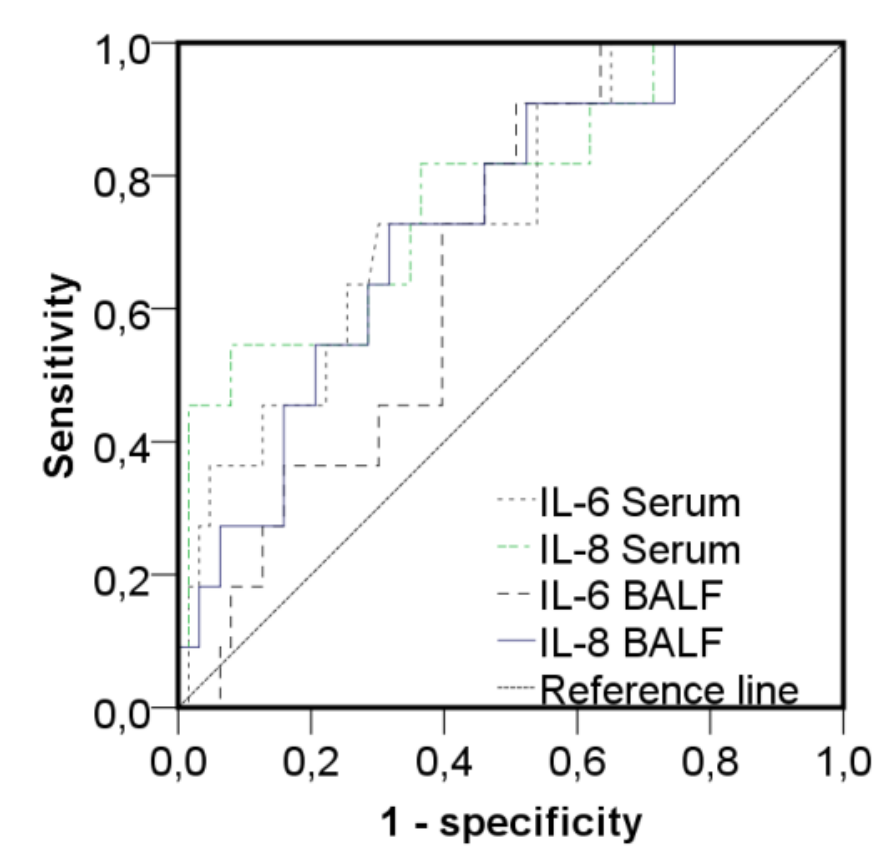

Table 1 (above): Performance of diagnostic tests in same-day serum and BALF samples, as well as combinations, for differentiating probable IA $(n=11)$ vs. cases with no evidence for IA $(n=63)$, ordered by DOR.

Table 2 (below):

Characteristics of cases with probable IA and no evidence for IA. \#: Included in cases without evidence for IA 4 CLL, 2 primary myelofibrosis, 2 Hodgkin's lymphoma and 1 anaplastic anemia. §: probable $\mathrm{IA}=1$ bacterial; no IA = 8 bacterial, 3 Pneumocystis, 1 Toxoplasma.

\begin{tabular}{|c|c|c|c|c|c|}
\hline $\begin{array}{c}\text { Test } / \\
\text { test combination }\end{array}$ & Sensitivity & Specificity & PPV & NPV & DOR \\
\hline $\begin{array}{c}\text { Serum IL-8 } \\
(>300 \mathrm{pg} / \mathrm{mL})\end{array}$ & $45 \%(5 / 11)$ & $98 \%(62 / 63)$ & $\begin{array}{l}83 \% \\
(5 / 6)\end{array}$ & \begin{tabular}{|c|}
$91 \%$ \\
$(62 / 68)$ \\
\end{tabular} & $\begin{array}{c}51.7 \\
(5.1-518) \\
\end{array}$ \\
\hline $\begin{array}{l}\text { Serum IL-8 } \\
(>60 \mathrm{pg} / \mathrm{mL})\end{array}$ & $55 \%(6 / 11)$ & $92 \%(58 / 63)$ & \begin{tabular}{|c|}
$55 \%$ \\
$(6 / 11)$ \\
\end{tabular} & $\begin{array}{c}92 \% \\
(58 / 63) \\
\end{array}$ & \begin{tabular}{|c|}
13.9 \\
$(3.1-62.2)$ \\
\end{tabular} \\
\hline $\begin{array}{l}\text { Serum IL-8 } \\
(>14 \mathrm{pg} / \mathrm{mL})\end{array}$ & $82 \%(9 / 11)$ & $63 \%(40 / 63)$ & \begin{tabular}{|c|}
$28 \%$ \\
$(9 / 32)$ \\
\end{tabular} & \begin{tabular}{|c|}
$95 \%$ \\
$(40 / 42)$ \\
\end{tabular} & \begin{tabular}{|c|}
7.8 \\
$(1.6-39.4)$ \\
\end{tabular} \\
\hline $\begin{array}{l}\text { Serum IL-6 } \\
(>40 \mathrm{pg} / \mathrm{mL})\end{array}$ & $73 \%(8 / 11)$ & $70 \%(44 / 63)$ & \begin{tabular}{c|}
$30 \%$ \\
$(8 / 27)$ \\
\end{tabular} & $\begin{array}{c}94 \% \\
(44 / 47) \\
\end{array}$ & \begin{tabular}{|c|}
6.2 \\
$(1.5-25.9)$ \\
\end{tabular} \\
\hline $\begin{array}{l}\text { Serum LFD } \\
\text { (45 min) }\end{array}$ & $9 \%(1 / 11)$ & $97 \%(61 / 63)$ & $\begin{array}{l}33 \% \\
(1 / 3)\end{array}$ & \begin{tabular}{|c|}
$86 \%$ \\
$(61 / 71)$ \\
\end{tabular} & \begin{tabular}{|c|}
3.0 \\
$(0.3-36.9)$ \\
\end{tabular} \\
\hline $\begin{array}{l}\text { Serum BDG } \\
(>80 \mathrm{pg} / \mathrm{mL}) \\
\end{array}$ & $45 \%(5 / 11)$ & $75 \%(47 / 63)$ & $\begin{array}{l}24 \% \\
(5 / 21) \\
\end{array}$ & $\begin{array}{c}89 \% \\
(47 / 53) \\
\end{array}$ & $\begin{array}{c}2.4 \\
(0.7-9.1) \\
\end{array}$ \\
\hline $\begin{array}{c}\text { Blood } \\
\text { Aspergillus PCR }\end{array}$ & $0 \%(0 / 10)$ & $100 \%(55 / 55)$ & - & $\begin{array}{c}85 \% \\
(55 / 65) \\
\end{array}$ & 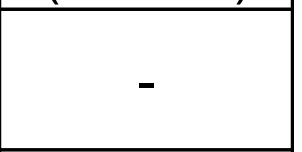 \\
\hline $\begin{array}{l}\text { Serum LFD } \\
(15 \mathrm{~min})\end{array}$ & $0 \%(0 / 10)$ & $98 \%(54 / 55)$ & - & $\begin{array}{c}84 \% \\
(54 / 64) \\
\end{array}$ & - \\
\hline $\begin{array}{l}\text { BALF LFD } \\
(10 \mathrm{~min}) \\
\end{array}$ & $73 \%(8 / 11)$ & $95 \%(60 / 63)$ & \begin{tabular}{|c|}
$73 \%$ \\
$(8 / 11)$ \\
\end{tabular} & $\begin{array}{c}95 \% \\
(60 / 63) \\
\end{array}$ & $\begin{array}{c}53.3 \\
(9.2-310) \\
\end{array}$ \\
\hline $\begin{array}{c}\text { BALF } \\
\text { Aspergillus PCR }\end{array}$ & $27 \%(3 / 11)$ & $98 \%(58 / 59)$ & $\begin{array}{l}75 \% \\
(3 / 4) \\
\end{array}$ & $\begin{array}{c}91 \% \\
(58 / 64) \\
\end{array}$ & \begin{tabular}{|c|}
21.8 \\
$(2.0-235)$ \\
\end{tabular} \\
\hline $\begin{array}{l}\text { BALF LFD } \\
\text { (15 min) }\end{array}$ & $73 \%(8 / 11)$ & $87 \%(55 / 63)$ & \begin{tabular}{|c|}
$50 \%$ \\
$(8 / 16)$ \\
\end{tabular} & $\begin{array}{c}95 \% \\
(55 / 58)\end{array}$ & \begin{tabular}{|c|}
18.3 \\
$(4.0-83.8)$
\end{tabular} \\
\hline $\begin{array}{c}\text { BALF IL-8 } \\
(>556 \mathrm{pg} / \mathrm{mL})\end{array}$ & $91 \%(10 / 11)$ & $48 \%(30 / 63)$ & \begin{tabular}{|c|}
$23 \%$ \\
$(10 / 43)$ \\
\end{tabular} & $\begin{array}{c}97 \% \\
(30 / 31) \\
\end{array}$ & \begin{tabular}{|c|}
9.1 \\
$(1.1-75.3)$ \\
\end{tabular} \\
\hline $\begin{array}{c}\text { BALF IL-8 } \\
(>1000 \mathrm{pg} / \mathrm{mL})\end{array}$ & $73 \%(8 / 11)$ & $67 \%(42 / 63)$ & \begin{tabular}{|c|}
$28 \%$ \\
$(8 / 29)$ \\
\end{tabular} & $\begin{array}{c}93 \% \\
(42 / 45)\end{array}$ & \begin{tabular}{|c|}
5.3 \\
$(1.3-22.2)$
\end{tabular} \\
\hline \begin{tabular}{|c|} 
Serum IL-8 \\
$(>300 \mathrm{pg} / \mathrm{mL})+/-$ \\
BALF LFD $(10 \mathrm{~min})$
\end{tabular} & $100 \%(11 / 11)$ & $94 \%(59 / 63)$ & $\begin{array}{c}73 \% \\
(11 / 15)\end{array}$ & \begin{tabular}{|c|}
$100 \%$ \\
$(59 / 59)$
\end{tabular} & $\begin{array}{c}304 \\
(15.3-6042)\end{array}$ \\
\hline \begin{tabular}{|c|} 
Serum IL-8 \\
$(>300 \mathrm{pg} / \mathrm{mL})+/-$ \\
BALF LFD $(15 \mathrm{~min})$
\end{tabular} & $100 \%(11 / 11)$ & $86 \%(54 / 63)$ & $\begin{array}{c}55 \% \\
(11 / 20)\end{array}$ & $\begin{array}{c}100 \% \\
(54 / 54)\end{array}$ & $\begin{array}{c}132 \\
(7.2-2432)\end{array}$ \\
\hline $\begin{array}{c}\text { Serum IL-8 } \\
(>300 \mathrm{pg} / \mathrm{mL})+/- \\
\text { BALF Asp. PCR }\end{array}$ & $91 \%(10 / 11)$ & $97 \%(57 / 59)$ & $\mid \begin{array}{c}83 \% \\
(10 / 12)\end{array}$ & $\left|\begin{array}{c}98 \% \\
(57 / 58)\end{array}\right|$ & $\begin{array}{c}285 \\
(23.6-3447)\end{array}$ \\
\hline
\end{tabular}

\begin{tabular}{|l|c|c|}
\hline & Probable IA (n=11) & No IA (n=63) \\
\hline $\begin{array}{l}\text { Female / Male } \\
\text { Age: Median (Range) }\end{array}$ & $\begin{array}{c}55 \%) / 5(45 \%) \\
34(54 \%) / 74)\end{array}$ & $57(27-85)$ \\
\hline AML or MDS & $6(50 \%)$ & $23(37 \%)$ \\
NHL & $2(18 \%)$ & $\begin{array}{c}18(29 \%) \\
8(13 \%)\end{array}$ \\
MM & $\begin{array}{c}1(9 \%) \\
2(18 \%)\end{array}$ & $\begin{array}{c}5(14 \%) \\
\text { ALL }\end{array}$ \\
\hline Others
\end{tabular}

Abbreviations: AF=antifungal; $A L L=$ acute lymphocytic leukemia; $A M L=a c u t e$ myelogenous leukemia; Asp.=Aspergillus; $A U C=$ area under the curve; $B A L F=$ bronchoalveolar lavage fluid; $B D G=\beta-D$ glucan; $D O R=$ diagnostic odds ratio; $G M=$ galactomannan; $G v H D=$ graft versus host disease; IA=invasive aspergillosis; IL=interleukin $I M I=$ invasive mold infection; $L F D=A s p e r g i l l u s$ specific lateral-flowdevice test; $M D S=$ myelodysplastic syndrome; $M M=$ multiple myeloma; $N H L=$ Non-Hodgkin lymphoma; $N P V=$ negative predictive value $P P V=$ positive predictive value; $\quad R O C=$ receiver operating characteristics; SCT=stem cell transplantation.

This work was supported by funds of the Gilead Investigator Initiated Study IN AT-131-1939, and the Oesterreichische Nationalbank (Anniversary Fund, project number 15346). Parts of the work have been carried out with the K1 COMET Competence Center CBmed. The funders had no role in study design, data collection, analysis, interpretation, decision to publish, in the writing of the manuscript, and in the decision to submit the manuscript for publication. 